\title{
Biological properties of andrographolide, an active ingredient of Andrographis Paniculata: a narrative review
}

\author{
Vaclav Vetvicka ${ }^{1}$, Luca Vannucci ${ }^{2}$ \\ ${ }^{1}$ Department of Pathology, University of Louisville, Louisville, KY, USA; ${ }^{2}$ Department of Immunology, Institute of Microbiology, Prague, Czech \\ Republic \\ Contributions: (I) Conception and design: Both authors; (II) Administrative support: V Vetvicka; (III) Collection and assembly of data: Both authors; (V) \\ Data analysis and interpretation: Both authors; (V) Manuscript writing: Both authors; (VI) Final approval of manuscript: Both authors. \\ Correspondence to: Vaclav Vetvicka. Department of Pathology, University of Louisville, Louisville, KY 40202, USA. \\ Email: vaclav.vetvicka@louisville.edu.
}

\begin{abstract}
Andrographolide is a labdane diterpenoid isolated from Andrographis paniculata and traditionally used in Chinese and Indian medicine. Reported effects include anti-bacterial, anti-inflammatory and anti-cancer functions. Most of the studies support the hypothesis that andrographolide supplementation stimulates immune system, so the observed effects migh in fact be secondary to the stimulation of defense reactions. As andrographolide is involved in regulation of inflammation, it is not surprising that it is also evaluated in inflammation-mediated diseases such as ulcerative colitis. Anticancer effects of the andrographolide have been tested on various cancer panels. Colon cancer, breast cancer, and head and neck carcinomas were the most investigated, followed by prostate cancer and glioblastoma. The results looked promising. However, problems with solubility and low level of active substance in natural extract leads to preparation of chemical analogs. Objective of this short review is to summarize current knowledge of the biological effects of andragrapholide. We conclude that despite documented effects and some partly characterized mechanisms of action, more research is clearly needed. At present, the doses, types of treatment and possible negative side effects are not yet established. In addition, various isolations and compound formulas have been used for treatment of various diseases, making final conclusions problematic.
\end{abstract}

Keywords: Andrographolide (AG); immunity; cancer; infection; signaling

Submitted Dec 05, 2020. Accepted for publication Feb 28, 2021.

doi: $10.21037 /$ atm-20-7830

View this article at: https://dx.doi.org/10.21037/atm-20-7830

\section{Introduction}

Andrographolide (AG) is a natural diterpenoid and represents the major biologically active ingredient of Andrographis paniculata. Among described effects are stimulation of the immune system (1), blocking the development of psoriasis and antibacterial and antiviral activities (2). The major biological effects are summarized in Figure 1. This results in its use in traditional Chinese and ayurvedic medicine. A pulmonary delivery of liposomal AG powder resulted in amelioration of bacterial pneumonia via downregulation of inflammation and inhibition of phosphorylation of the $\mathrm{I} \kappa \mathrm{B} \alpha$ in the NF- $\kappa \mathrm{B}$ pathway (3).
Molecular mechanisms of AG action are still unclear. Most of the studies reported suppression of NF-kB activation. However, others described changes in PI3K and MAPK pathways, and inhibition of NFAT activation (4).

Despite repeatedly demonstrated significant biological activity, further use of AG in pharmacology and medicine is hindered by its poor selectivity and low solubility, leading to attempts to modify its structure and formation of several analogs. Low levels of AG in various extracts has been lately improved by use of different inducers (5), but the results are not satisfactory thus far.

In this review, we focused on the most documented and most promising aspects of the use of AG in several health 


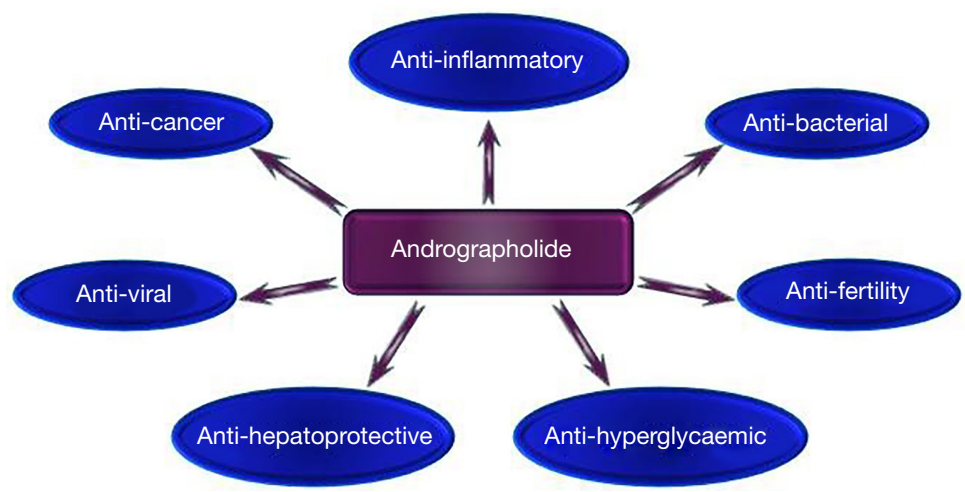

Figure 1 Major biological effects of andrographolides.

problems. We present the following article in accordance with the Narrative Review reporting checklist (available at https://dx.doi.org/10.21037/atm-20-7830).

\section{Methods}

An English language literature search utilizing the PubMed for years 2000 to 2020 was used. Collected data were based on keywords including AG, inflammation, cancer, immunity, infection and medicine.

\section{Inflammation}

AG and its derivatives have some anti-inflammatory activity. The study of possible mechanisms of AG action focused on the interaction with TLRs. The investigation confirmed that AG suppressed NF- $\mathrm{KB}$ activation and COX2 expression caused by signaling via TLR3 and TLR4 by targeting TBK1 (6). The strength and distinction of these effects makes AG a promising candidate for antiinflammatory treatment.

When tested on an experimentally induced murine colitis, this reduction of inflammation resulted in improvement of the disease and even reversed the pathology (7). The possible mechanisms of action include secretion reduction of several inflammatory cytokines including IL-6 and IFN- $\gamma$. Based on computational chemistry, AG has at least three different mechanisms of anti-inflammatory activity including inhibition of production of oxygen radicals, suppression of inducible iNOS expression and inhibition of NF-kB (8).

Most studies describing activities of Andrographis paniculata extracts focused on andragrapholide. However, additional components such as dehydroxyandrographolide and neoandrographolide also exert some biological effects, most of all interactions with inflammatory processes [for review see Parichatikanond et al. (9)]. Direct evaluation of anti-inflammatory activities of all three types of Andrographis paniculata extracts revealed all three inhibit inflammation via interfering COX and inflammatory cytokines. AG has an additional effect in downregulating the expression of some genes participating in inflammatory processes (9).

As AG is involved in regulation of inflammation, it is not surprising that it is also evaluated in inflammation-mediated diseases such as ulcerative colitis. Continuous delivery of AG resulted in significant decrease of pro-inflammatory cytokines IL-1 $\beta$, TNF- $\alpha$, IL- 6 and IL-17A. In addition, a reduction in numbers of Th17 cells in colon tissue was found. The authors concluded that AG supplementation decreased Th17 immune response, probably due to the inhibition of STAT3 signaling (10). Using a similar model of experimental colitis, Jing and co-workers confirmed palliative effects of AG, but found different mechanisms. Their in vitro and in vivo experiments showed that AG inhibited the expression of phosphorylated NF- $\kappa \mathrm{B}, \mathrm{I} \kappa \mathrm{B} \alpha$, and p65, lowered expression of COX-2 and iNOS and inhibited activation of MAPK signaling (11).

\section{Infection}

AG has been used for centuries in treatment of infections. The mechanisms are not completely known, and it might vary in different types of infections. In case of Pseudomonas aeruginosa, it seems that AG has multiple effects. Firstly, is the action oriented towards a QS system, resulting in inhibition of biofilm formation via lowering the expression 


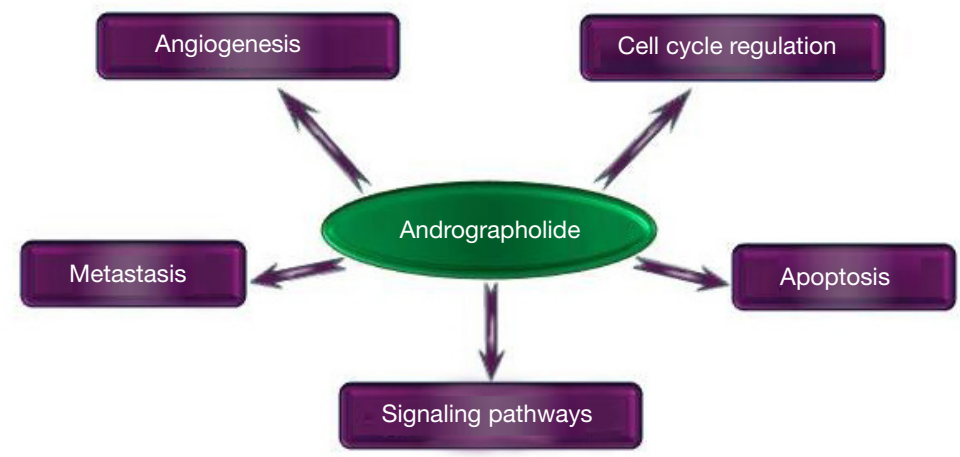

Figure 2 Suggested mechanisms of action.

of Psl polysaccharides (12). Secondary, effects are manifested by inhibition of virulence factors by production obstruction of numerous virulence factors such as pyocyanin (13). Repression of the MexAB-OprM exhaust pump by AG lowers the multidrug resistance (14). Different action has been found in Staphylococcus aureus infection. The major action of AG in protection against this type of infection is the result of regulation of immune reactions. AG can reduce inflammatory reaction via inhibition of formation of inflammatory cytokines such as IL-6 and TNF- $\alpha$ and by expression blockage of apoptotic proteins and NF-kB activation (15). Additional mechanisms involve suppression of the transcriptional regulator SarA, which subsequently reduces the formation of biofilm and expression of various virulent factors (16). In Escherichia coli infection, AG blocks Autoinducer-2, which is a signaling molecule involved in inter- and intra-species communication (17). In addition, AG can lower the bacterial adhesion to the cells (18). Furthermore, AG can damage the integrity of the bacteria directly, probably via damages of the cytoskeleton. For more details about AG effects and its analogs on infection agents, see recent review Zhang et al. (19).

An interesting study evaluated the possible effects of increased solubility of AG on its biological results. The authors found significant anti-inflammatory effects and higher phagocytosis by macrophages. When tested for anti-bacterial effects, only very limited activity was found in vitro, but using an in vivo model, significant improvements in survival have been found. The authors speculate that AG can protect against infection via stimulation of immunity (20). The quality of this study is decreased, however, by omitting the normal AG, making the conclusions about possible improvement via increased solubility impossible.
AG was also tested as a possible anti-viroticum. Using an experimental model of in vitro enterovirus D68 infection, AG was found to inhibit viral replication and protein synthesis. Later experiments suggested that this suppression is mediated via changes in the maturation of virus-containing endosomes, particularly by prevention of acidification of endosomes (21).

\section{Cancer}

Diterpenoids are known to possess a quite wide range of biological effects and represent an interesting category of molecules suitable even for possible oncological treatments. Anticancer effects of the diterpenoid AG have been tested on various cancer panels. Colon cancer, breast cancer, and head and neck carcinomas were the most investigated, followed by prostate cancer and glioblastoma. A systematic review of over 1,703 articles on AG and cancer found that AG has significant anticancer effects on all types of cancer tested (22). With over 15 suggested mechanisms described in these studies (Figure 2), the real mechanisms of action are currently almost impossible to pinpoint but, clearly, AG reflects a promising tool in our fight against cancer.

Neoplasms still represent a relevant problem in their management since their variability in response to the anticancer treatment that, in almost half of the cases, starts in the presence of an already spread disease. The necessity to improve efficacy of treatment and quality of life with interventions having low toxicity and possible enhancement of standard therapeutic effects may find an answer in the derivatives of natural products. Research on the antitumor efficacy of AG has revealed that this molecule can exert interesting pro-apoptotic and anti-inflammatory effects. 
It may also be a possible enhancer of radio- and chemosensitivity in the treated tumors.

In a study by Lee et al. (23), the authors used RAW264.7 macrophages stimulated with LPS. Results showed that AG was able to inhibit the activated inflammatory signaling pathway. Decrease of iNOS and COX-2 protein levels with consequent PGE2 reduction were documented. ROS downregulation was inhibiting NF- $\kappa \mathrm{B}$ and $\mathrm{AP}-1$ expression. Decrease of STAT3 phosphorylation, SOCS1 and SOCS3 expression were also found. All these events affected the production of IL- $1 \beta$, IL- 6 , and TNF- $\alpha$, indicating the relevant potential of AG as an anti-inflammatory molecule. The possibility to control inflammatory processes impacting tumor establishment and progression is known from the many studies on tumor microenvironment immunology, with particular interest for head and neck carcinomas and, perhaps, glioblastoma (24-27). The tests with AG performed on these tumors appear promising for controlling the tumor growth.

Nasopharyngeal carcinoma cells, 7,12-dimethyl-1,2benzanthracene (DMBA) induced oral squamous cell carcinoma in the hamster model of cheek buccal pouch and carcinoma of the tongue cells. This shows the AG treatment was able to inhibit NF- $\mathrm{kB}$. Using a nasopharyngeal carcinoma cell line, AG was found to inhibit proliferation and induce apoptosis. The study also found changes in regulation of the LKB1/AMPK/mTOR signaling pathway, but it is not clear if this regulation really caused the changes in cancer cell proliferation (28). In the in vivo model, the dyterpenoid reduced the phosphorylation of $\mathrm{p} 65$ and $\mathrm{I \kappa B} \alpha$ with consequent block of the NF- $\mathrm{KB}$ aberrant activation but also exerted a negative role on angiogenesis. In the in vitro model, suppressive activity on NF- $\mathrm{BB}$ target genes EGFR, cyclin D1 and survivin. Also, genes regulating proliferation, angiogenesis and migration were negatively affected (MMP-9, ICAM-1, c-Myc, and VEGF), promoting cell apoptosis (27). Similar effects on COX-2 and angiogenesis were found in breast cancer (29) and insulinoma (by interfering with the TLR4 pathway) (30). The anti-angiogenetic properties of AG, tested in multiple models of vascular development (like chick embryo chorioallantoic membrane and yolk sac membrane angiogenesis models and HUVEC cell tube formation), were also found depending on inhibition of the miR-21-5p/TIMP3 signaling pathway (31).

Two other main effects of AG are the inhibition of cell replication and migration. When Andrographis paniculata extract was further fractionated into aqueous, methane and ether fractions, three were found to inhibit the proliferation of cancer cells. The only non-active fraction was the aqueous one. Simultaneously, these fractions elevated proliferation of peripheral blood lymphocytes and induced IL-2 formation (32). This suggests simultaneous anticancer and immunomodulatory activities of AG. Breast MDAMB-231, MCF-7, and HCT-116 cancer cells demonstrated a replication arrest in G1 $(33,34)$, same in colon cancer cells. Another study showed the arrest in G2/M for the HT29 colon cancer cells but also revealed that, depending on the doses, the low dose treatment was producing the G1 arrest. Higher dosages were arresting the replication in G1/ G0 (35). Even in the cancer cell models of liver (HepG2 and SK-Hep1) and bile duct (HuCCA-1 and RMCCA-1) the proliferation block was described as depending on the arrest in G0/G1 and G2/M phases (36). In this tumor, the treatment reduced the expression of cyclin-D1, Bcl-2 and the inactive proenzyme form of caspase- 3 and increase of the pro-apoptotic protein Bax. Antiproliferative activity on colon cancer cells (SW-480) was also associated to downregulation of the Notch pathway (37). One of the proposed mechanisms of action is summarized in Figure 3.

Inhibition to migration was documented in the glioblastoma cell model where motility was impeded by downregulation of the ERK1/2-mediated matrix metalloproteinase-2 (MMP-2) expression $(39,40)$. This is important as an anti-metastatic effect of AG described for Ras-transformed cells, like the glioblastoma (41). Similar anti-metastatic downregulation of MMP-2, associated also to MMP-9, was found to be induced also in gastric cancer cells (SGC7901). In these cells, the cell cycle arrest in G2/ M2 or G1/M (according to the dose of AG and apoptosis consequent to increase of Timp-1/2, cyclin B1, p-Cdc2, Bax and reduction of Bcl-2) was reported (42). Apoptosis is a very common effect of the treatment, observed in many different tumor models (breast cancer, intrahepatic cholangiocarcinoma, colon HNCC, osteosarcoma glioblastoma, prostate cancer, human T-cell acute lymphoblastic leukemia Jurkat cells, bladder cancer, gastric carcinoma) (33-36,41-49). In all models, the apoptosis appears to be a consequence of ROS/JNK pathway or ERK-p53 with caspases activation, PI3K-AKT-p53 signaling pathway, endoplasmic reticulum stress associated to IRE-1 signaling or mitochondrial damage. The increase of ROS levels appears to be the most common apoptotic mechanism induced in colon cancer by the $\mathrm{AG}(35,44,46)$.

Another interesting aspect of AG is the capability to act as a therapeutic enhancer of radio- and chemotherapy. Oral carcinoma increased the expression of microRNA-218, 


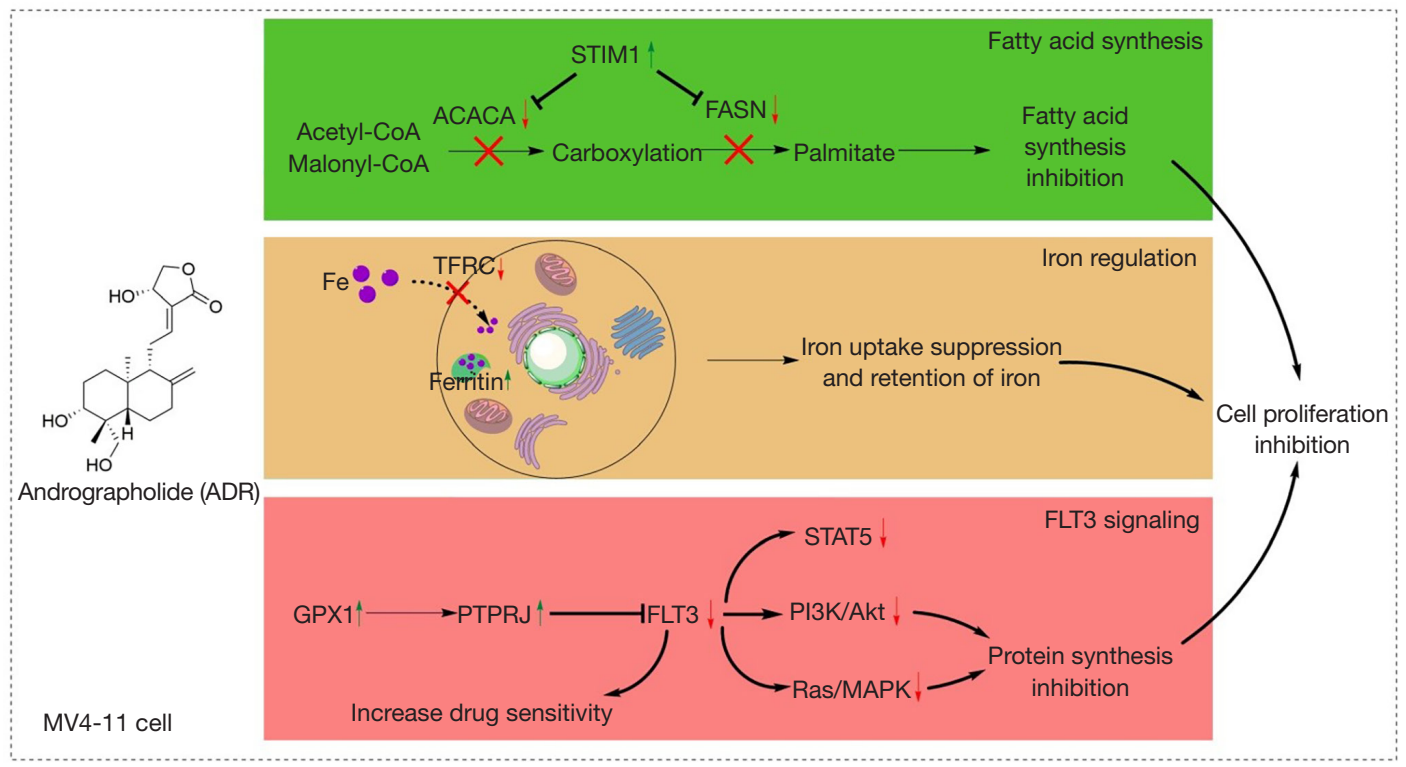

Figure 3 Proposed mechanism for the proliferation inhibitory effects of ADR in MV4-11 cells. From (38).

leading to the downregulation of Bmi1 with impediment to the cancer stemness (50). Radiosensitivity is increased also by the inhibition of ERK-mediated MMP-2 activity (51) in the model of Ras-transformed cells (e.g., glioblastoma). The pro-apoptotic activity of vincristine and carbo-platinum in HNCC, through increase of ROS, are an example of chemosensitization finally affecting the AKT-p53 pathway (52-54). Sensitization of colon cancer cells to 5 -fluorouracil $(5-\mathrm{FU})$ is mediated by reduction of the p-MET level in the combination treatment (55). Finally, two possible new applications for AG were proposed recently, the first is the preparation of nanofibrous membranes, biodegradable and locally delivering AG for the treatment of cervical cancer (56). The second is the evidence of a protective action on the gut mucosa by AG through regulation of the p38 MAPK pathway (reduction of caspase8/3, Bax and the phosphorylation of p38) (57). Therefore, while sensitizing the tumor to the 5-FU action, the bowel can produce an anti-mucositis effect on the mucosa.

Recently, AG has shown to have anti-proliferative effects in in vitro experiments involving induction of apoptosis by activation of pro-apoptotic Bcl2 family in cancer cells (15). Detailed research revealed AG induced mitochondrial-mediated apoptosis in several lymphoma cells with significant involvement of caspases. These effects were blocked by addition of known antioxidant $\mathrm{N}$-acetylL-cysteine. Similar results were obtained in cases of human colon cancer cells, where AG induced apoptosis (42). All these results have been obtained only in vitro, so their clinical value is limited. However, the findings of synergistic effects of an AG-5-fluorouracil combination are interesting.

Some effects of AG on cancer development might be caused by stimulation of NK (58) and ADCC cell activity, antibody-dependent complement-mediated cytotoxicity and by inhibition of secretion of pro-inflammatory cytokines (TNF- $\alpha$, IL-1 $\beta$, and IL-6). It was surprising that the crude extract of Andrographis paniculata showed stronger effects than isolated AG $(59,60)$, suggesting the biological activity of yet unknown molecules present in the extract.

Further development of AG as a potential drug is limited to its pharmacological setbacks, leading to preparation of numerous analogs. For a review of anti-cancer effects of these analogs with novel anti-cancer effects, see Soo et al. (61).

\section{Immunity}

Most of the studies support the hypothesis that AG supplementation stimulates immune system. However, one group found that AG can regulate T-cell activation and down-regulate both cellular and humoral branches of immunity (62). The same group later found that interfering with NF-kB pathway by AG changed the capacity of dendritic cells to induce tolerance in mice with autoimmune encephalomyelitis (63). If confirmed, this study might open 
a new window for the therapeutic use of AG for inhibiting development of autoimmune disease by elevating the antigen-specific tolerance.

Immunomodulatory activity of AG showed increased antibody response, stimulation of phagocytosis and improved cellularity in the spleen and thymus after suppression caused by application of cyclophosphamide (64). However, the quality of this study is rather low due to the use of an extract formed by three different AGs and using obsolete techniques such as plaque assay, making any significant conclusions rather difficult.

In vitro addition of $\mathrm{AG}$ resulted in reduction of macrophage activation and inhibition of M1/M2 polarization. Supplementation with $1 \mathrm{mg} / \mathrm{kg}$ AG caused significant suppression of antibody response. Some data also suggested possible participation of PI3K and MAPK signaling pathways (65). These immunomodulatory effects confirmed that AG modulates both natural and adaptive branch of immune reactions by affecting both macrophages and lymphocytes.

To better approach the mechanisms of action, several AG derivatives were synthesized and analyzed. The results indicated production suppression of pro-inflammatory cytokines via activation of TLR3 and TLR4 ligands. Not all derivatives produced the same effects, some inhibited nuclear translocation of NF- $\mathrm{KB}$ p 65 subunit. On one hand, the study confirmed significant biological effects of AG on innate immunity, on the other hand demonstrated strong relation between AG structure and its function with only one out of 18 derivatives being recommended for further development (66).

Experiments focused on antibody response effects found that both Andrographis paniculata extract and isolated AG have same positive results on elevated antibody formation after Salmonella vaccine application. Subsequent in vitro experiments showed elevated production of INF- $\gamma$ by spleen cells from vaccinated animals, showing that both humoral and cellular response has been raised (67).

\section{Other effects}

Numerous experiments demonstrated that AG crosses the blood-brain barrier and moves into different regions of the brain. This discovery led to subsequent studies of the effects on the CNS. Double-blind clinical trials of longterm supplementation with $\mathrm{AG}$, in patients with non-active progressive multiple sclerosis, found potential positive effects on brain atrophy and disease progression (68), but larger studies are needed to confirm these findings. An interesting approach showed new avenues for treatment development of neurological disorders including memory impairment. In vitro experiments revealed AG inhibited experimentally induced overexpression of COX-2, NF-кB, TLR4, and iNOS. In addition, release of inflammatory mediators was inhibited both in vitro in primary glial cell cultures and in vivo in mouse prefrontal cortex. Further experiments suggested improvements of memory and neuron protection via genomic changes and neuroinflammation suppression (69).

In an animal model, AG was found to improve middle cerebral artery occlusion and brain infarct (70). Among suggested mechanisms, the most promising seems to be the results on endothelial cells and improvement of brain homeostasis [for review see Lu et al. (71)]. The other effects are more speculative and involve effects of AG on platelets resulting in improvements of blood supply to the brain. AG supplementation was also found in Parkinson disease, Alzheimer disease and intracerebral hemorrhage, but the action mechanisms are unknown (71). However, the outcomes of AG on CNS are strong enough that they resulted in several currently running clinical trials, often focused on severe anti-depressant-like activities.

AG was found to have hypoglycemic effects in mouse model of diabetes type 1 , probably via inhibition of NF- $\mathrm{KB}$ pathway (72). A different model of experimental diabetes induced by streptozotocin also found lowering of plasma glucose levels by AG supplementation (73). Subsequent studies suggested that 4-week oral supplementation with AG manifested preventive effects on the development and progression of diabetes. Based on production changes of several cytokines (such as IL-2, IL-10 and IFN- $\gamma$ ) and changes in expression of some mRNA (such as T-bet and GATA3), the authors concluded these preventive effects are mediated via maintaining of Th1/Th2/Th17 homeostasis (74) resulting in protection of $\beta$ cells. A comprehensive review of possible effects of AG on metabolic treatment suggested promising opportunities, but warned that our recent knowledge is not yet adequate (22).

Another possible role of AG involves protection against lung injury. Using an ovalbumin-induced lung injury model, Peng et al., showed that AG application significantly reduced lung injury by inhibition of ROS-mediated $\mathrm{NF}-\kappa \mathrm{B}$ signaling and activation suppression of NLRP3 inflammasome (75). If confirmed, AG might be developed into a new way to treat asthma. A sulfonated version, AG-S, has been shown to have even stronger effects. In addition, 
it is fully soluble and has been widely used for various treatments. A detailed proteomic study showed that AG-S inhibits lung injury via anti-inflammatory mechanisms including suppression of myeloperoxidase and neutrophilderived proteases (76).

Due to its anti-inflammatory effects, AG was also evaluated as a possible treatment in rheumatoid arthritis. Several studies found significant effects in reduction of serological parameters of the disease and in relief of various symptoms [for review see Hidalgo et al. (77)] via reduction of the activation of various transcriptional factors such as AP-1, NFAT, STAT3, and NF-кB.

Much less studied are additional biological effects of AG. Intraperitoneal application of AG into mice, with experimentally induced diabetic retinopathy, showed significant amelioration of this disease by attenuation of retinal inflammation and angiogenesis. In addition, changes in VEGF, NF-кB, and Erg1 signaling pathways were found to be involved (78).

For a summary of additional potential effects, including anti-malaria activity, anti-allergy reactions, cardiovascular protection, hepatic protection, and suppression of skin diseases, see an excellent overview by Dai et al. (79).

\section{Conclusions}

AG is an extract of Andrographis paniculata, traditional Chinese medicinal plant, with various significant biological effects. Among the most pronounced activities are antibacterial and antitumor effects. Significant stimulation and regulation of the immune reactions represent another well-documented biological activity of AG. Based on the recent findings, AG and particularly its analogs, offer a new window for further pharmacological investigations leading to improve our ways of treatment of numerous diseases including cancer and infections. However, more clinical and experimental research is necessary to fully establish the doses, types of treatment and possible negative side effects. In addition, various isolations and compound formulas have been used for treatment of various diseases, making final conclusions problematic. Further research on this molecule to assess its full potential to become a drug is needed.

\section{Acknowledgments}

Funding: The authors would like to acknowledge the financial support of the RVO 61388971 grant.

\section{Footnote}

Reporting Checklist: The authors have completed the Narrative Review reporting checklist. Available at https:// dx.doi.org/10.21037/atm-20-7830

Conflicts of Interest: Both authors have completed the ICMJE uniform disclosure form (available at https://dx.doi. org/10.21037/atm-20-7830). Dr. Vetvicka serves as an unpaid editorial board member of Annals of Translational Medicine from Jan 2020 to Dec 2021. The other author has no conflicts of interest to declare.

Ethical Statement: The authors are accountable for all aspects of the work in ensuring that questions related to the accuracy or integrity of any part of the work are appropriately investigated and resolved.

Open Access Statement: This is an Open Access article distributed in accordance with the Creative Commons Attribution-NonCommercial-NoDerivs 4.0 International License (CC BY-NC-ND 4.0), which permits the noncommercial replication and distribution of the article with the strict proviso that no changes or edits are made and the original work is properly cited (including links to both the formal publication through the relevant DOI and the license). See: https://creativecommons.org/licenses/by-nc-nd/4.0/.

\section{References}

1. Singha PK, Roy S, Dey S. Antimicrobial activity of Andrographis paniculata. Fitoterapia 2003;74:692-4.

2. Panraksa $\mathrm{P}$, Ramphan $\mathrm{S}$, Khongwichit $\mathrm{S}$, et al. Activity of andrographolide against dengue virus. Antiviral Res 2017;139:69-78.

3. Li M, Zhang T, Zhu L, et al. Liposomal andrographolide dry powder inhalers for treatment of bacterial pneumonia via anti-inflammatory pathway. Int J Pharm 2017;528:163-71.

4. Carretta MD, Alarcón P, Jara E, et al. Andrographolide reduces IL-2 production in T-cells by interfering with NFAT and MAPK activation. Eur J Pharmacol 2009;602:413-21.

5. Pandey G, Rao CH. Andrographolide: its pharmacology, natural bioavailability and current approaches to increase its content in Andrographis paniculate. Int J Complement Alt Med 2018;11:355-60.

6. Kim AY, Shim HJ, Shin HM, et al. Andrographolide 
suppresses TRIF-dependent signaling of toll-like receptors by targeting TBK1. Int Immunopharmacol 2018;57:172-80.

7. Guo BJ, Liu Z, Ding MY, et al. Andrographolide derivative ameliorates dextran sulfate sodium-induced experimental colitis in mice. Biochem Pharmacol 2019;163:416-24.

8. Levita J, Nawawi A, Mutalib A, et al. Andrographolide: A review of its anti-inflammatory activity via inhibition of NF-kappaB activation from computational chemistry aspects. Int J Pharm 2010;6:569-76.

9. Parichatikanond W, Suthisisang C, Dhepakson P, et al. Study of anti-inflammatory activities of the pure compounds from Andrographis paniculata (burm. f.) Nees and their effects on gene expression. Int Immunopharmacol 2010;10:1361-73.

10. Zhu Q, Zheng P, Chen X, et al. Andrographolide presents therapeutic effect on ulcerative colitis through the inhibition of IL-23/IL-17 axis. Am J Transl Res 2018;10:465-73.

11. Jing M, Wang Y, Xu L. Andrographolide Derivative AL-1 Ameliorates Dextran Sodium SulfateInduced Murine Colitis by Inhibiting NF- $\mathrm{KB}$ and MAPK Signaling Pathways. Oxid Med Cell Longev 2019;2019:6138723.

12. Ma L, Liu X, Liang H, et al. Effects of 14-alpha-lipoyl andrographolide on quorum sensing in Pseudomonas aeruginosa. Antimicrob Agents Chemother 2012;56:6088-94.

13. Richards JJ, Ballard TE, Huigens RW 3rd, et al. Synthesis and screening of an oroidin library against Pseudomonas aeruginosa biofilms. Chembiochem 2008;9:1267-79.

14. Wu CM, Cao JL, Zheng MH, et al. Effect and mechanism of andrographolide on the recovery of Pseudomonas aeruginosa susceptibility to several antibiotics. J Int Med Res 2008;36:178-86.

15. Cheung HY, Cheung SH, Li J, et al. Andrographolide isolated from Andrographis paniculata induces cell cycle arrest and mitochondrial-mediated apoptosis in human leukemic HL-60 cells. Planta Med 2005;71:1106-11.

16. Park JH, Lee JH, Kim CJ, et al. Extracellular protease in Actinomycetes culture supernatants inhibits and detaches Staphylococcus aureus biofilm formation. Biotechnol Lett 2012;34:655-61.

17. Lazazzera BA. Quorum sensing and starvation: signals for entry into stationary phase. Curr Opin Microbiol 2000;3:177-82.
18. Guo X, Zhang LY, Wu SC, et al. Andrographolide interferes quorum sensing to reduce cell damage caused by avian pathogenic Escherichia coli. Vet Microbiol 2014;174:496-503.

19. Zhang L, Bao M, Liu B, et al. Effect of Andrographolide and Its Analogs on Bacterial Infection: A Review. Pharmacology 2020;105:123-34.

20. Wen L, Xia N, Chen X, et al. Activity of antibacterial, antiviral, anti-inflammatory in compounds andrographolide salt. Eur J Pharmacol 2014;740:421-7.

21. Wang D, Guo H, Chang J, et al. Andrographolide Prevents EV-D68 Replication by Inhibiting the Acidification of Virus-Containing Endocytic Vesicles. Front Microbiol 2018;9:2407.

22. Islam MT. Andrographolide, a New Hope in the Prevention and Treatment of Metabolic Syndrome. Front Pharmacol 2017;8:571.

23. Lee KC, Chang HH, Chung YH, et al. Andrographolide acts as an anti-inflammatory agent in LPS-stimulated RAW264.7 macrophages by inhibiting STAT3-mediated suppression of the NF- $\mathrm{KB}$ pathway. J Ethnopharmacol 2011;135:678-84.

24. Gupta SC, Kunnumakkara AB, Aggarwal S, et al. Inflammation, a Double-Edge Sword for Cancer and Other Age-Related Diseases. Front Immunol 2018;9:2160.

25. Zhang Y, Kong W, Jiang J. Prevention and treatment of cancer targeting chronic inflammation: research progress, potential agents, clinical studies and mechanisms. Sci China Life Sci 2017;60:601-16.

26. Bonomi M, Patsias A, Posner M, et al. The role of inflammation in head and neck cancer. Adv Exp Med Biol 2014;816:107-27.

27. Sowers JL, Johnson KM, Conrad C, et al. The role of inflammation in brain cancer. Adv Exp Med Biol 2014;816:75-105.

28. Wu B, Chen X, Zhou Y, et al. Andrographolide inhibits proliferation and induces apoptosis of nasopharyngeal carcinoma cell line C666-1 through LKB1-AMPK-dependent signaling pathways. Pharmazie 2018;73:594-7.

29. Peng T, Hu M, Wu TT, et al. Andrographolide suppresses proliferation of nasopharyngeal carcinoma cells via attenuating NF- $\kappa$ B pathway. Biomed Res Int 2015;2015:735056.

30. Zhang QQ, Ding Y, Lei Y, et al. Andrographolide suppress tumor growth by inhibiting TLR4/NF$\kappa \mathrm{B}$ signaling activation in insulinoma. Int J Biol Sci 
2014;10:404-14.

31. Dai J, Lin Y, Duan Y, et al. Andrographolide Inhibits Angiogenesis by Inhibiting the Mir-21-5p/TIMP3 Signaling Pathway. Int J Biol Sci 2017;13:660-8.

32. Kumar RA, Sridevi K, Kumar NV, et al. Anticancer and immunostimulatory compounds from Andrographis paniculata. J Ethnopharmacol 2004;92:291-5.

33. Jada SR, Matthews C, Saad MS, et al. Benzylidene derivatives of andrographolide inhibit growth of breast and colon cancer cells in vitro by inducing $G(1)$ arrest and apoptosis. Br J Pharmacol 2008;155:641-54.

34. Banerjee M, Chattopadhyay S, Choudhuri T, et al. Cytotoxicity and cell cycle arrest induced by andrographolide lead to programmed cell death of MDA-MB-231 breast cancer cell line. J Biomed Sci 2016;23:40.

35. Khan I, Khan F, Farooqui A, et al. Andrographolide Exhibits Anticancer Potential Against Human Colon Cancer Cells by Inducing Cell Cycle Arrest and Programmed Cell Death via Augmentation of Intracellular Reactive Oxygen Species Level. Nutr Cancer 2018;70:787-803.

36. Suriyo T, Pholphana N, Rangkadilok N, et al. Andrographis paniculata extracts and major constituent diterpenoids inhibit growth of intrahepatic cholangiocarcinoma cells by inducing cell cycle arrest and apoptosis. Planta Med 2014;80:533-43.

37. Khan I, Mahfooz S, Saeed M, et al. Andrographolide Inhibits Proliferation of Colon Cancer SW-480 Cells via Downregulating Notch Signaling Pathway. Anticancer Agents Med Chem 2021;21:487-97.

38. Chen X, Zhang J, Yuan L, et al. Andrographolide Suppresses MV4-11 Cell Proliferation through the Inhibition of FLT3 Signaling, Fatty Acid Synthesis and Cellular Iron Uptake. Molecules 2017;22:1444.

39. Chiu SP, Batsaikhan B, Huang HM, et al. Application of Electric Cell-Substrate Impedance Sensing to Investigate the Cytotoxic Effects of Andrographolide on U-87 MG Glioblastoma Cell Migration and Apoptosis. Sensors (Basel) 2019;19:2275.

40. Yang SL, Kuo FH, Chen PN, et al. Andrographolide suppresses the migratory ability of human glioblastoma multiforme cells by targeting ERK1/2-mediated matrix metalloproteinase-2 expression. Oncotarget 2017;8:105860-72.

41. Dai L, Wang G, Pan W. Andrographolide Inhibits Proliferation and Metastasis of SGC7901 Gastric Cancer Cells. Biomed Res Int 2017;2017:6242103.
42. Khan I, Mahfooz S, Ansari IA. Antiproliferative and Apoptotic Properties of Andrographolide Against Human Colon Cancer DLD1 Cell Line. Endocr Metab Immune Disord Drug Targets 2020;20:930-42.

43. Banerjee A, Banerjee V, Czinn S, et al. Increased reactive oxygen species levels cause ER stress and cytotoxicity in andrographolide treated colon cancer cells. Oncotarget 2017;8:26142-53.

44. Bundela S, Sharma A, Bisen PS. Potential Compounds for Oral Cancer Treatment: Resveratrol, Nimbolide, Lovastatin, Bortezomib, Vorinostat, Berberine, Pterostilbene, Deguelin, Andrographolide, and Colchicine. PLoS One 2015;10:e0141719.

45. Wang S, Li H, Chen S, et al. Andrographolide induces apoptosis in human osteosarcoma cells via the ROS/ JNK pathway. Int J Oncol 2020;56:1417-28.

46. Yang SH, Wang SM, Syu JP, et al. Andrographolide induces apoptosis of C6 glioma cells via the ERKp53-caspase 7-PARP pathway. Biomed Res Int 2014;2014:312847.

47. Wei RJ, Zhang XS, He DL. Andrographolide sensitizes prostate cancer cells to TRAIL-induced apoptosis. Asian J Androl 2018;20:200-4.

48. Yang T, Yao S, Zhang X, et al. Andrographolide inhibits growth of human T-cell acute lymphoblastic leukemia Jurkat cells by downregulation of PI3K/AKT and upregulation of p38 MAPK pathways. Drug Des Devel Ther 2016;10:1389-97.

49. Deng Y, Bi R, Guo H, et al. Andrographolide Enhances TRAIL-Induced Apoptosis via p53-Mediated Death Receptors Up-Regulation and Suppression of the NF-кB Pathway in Bladder Cancer Cells. Int J Biol Sci 2019;15:688-700.

50. Yang PY, Hsieh PL, Wang TH, et al. Andrographolide impedes cancer stemness and enhances radio-sensitivity in oral carcinomas via miR-218 activation. Oncotarget 2017;8:4196-207.

51. Lu WJ, Lin KH, Hsu MJ, et al. Suppression of NF-кB signaling by andrographolide with a novel mechanism in human platelets: regulatory roles of the p38 MAPKhydroxyl radical-ERK2 cascade. Biochem Pharmacol 2012;84:914-24.

52. Yu CC, Chen CA, Fu SL, et al. Andrographolide enhances the anti-metastatic effect of radiation in Rastransformed cells via suppression of ERK-mediated MMP-2 activity. PLoS One 2018;13:e0205666.

53. Zhang $M$, Xue E, Shao W. Andrographolide promotes vincristine-induced SK-NEP-1 tumor cell death via 
PI3K-AKT-p53 signaling pathway. Drug Des Devel Ther 2016;10:3143-52.

54. Mao W, He P, Wang W, et al. Andrographolide sensitizes Hep-2 human laryngeal cancer cells to carboplatin-induced apoptosis by increasing reactive oxygen species levels. Anticancer Drugs 2019;30:e0774.

55. Su M, Qin B, Liu F, et al. Andrographolide enhanced 5-fluorouracil-induced antitumor effect in colorectal cancer via inhibition of c-MET pathway. Drug Des Devel Ther 2017;11:3333-41.

56. Chen YP, Liu YW, Lee D, et al. Biodegradable andrographolide-eluting nanofibrous membranes for the treatment of cervical cancer. Int J Nanomedicine 2019;14:421-9.

57. Xiang DC, Yang JY, Xu YJ, et al. Protective effect of Andrographolide on 5-Fu induced intestinal mucositis by regulating p38 MAPK signaling pathway. Life Sci 2020;252:117612.

58. Gong C, Ni Z, Yao C, et al. A High-Throughput Assay for Screening of Natural Products that Enhanced Tumoricidal Activity of NK Cells. Biol Proced Online 2015;17:12.

59. Sheeja K, Kuttan G. Andrographis paniculata downregulates proinflammatory cytokine production and augments cell mediated immune response in metastatic tumor-bearing mice. Asian Pac J Cancer Prev 2010;11:723-9.

60. Sheeja K, Kuttan G. Modulation of natural killer cell activity, antibody-dependent cellular cytotoxicity, and antibody-dependent complement-mediated cytotoxicity by andrographolide in normal and Ehrlich ascites carcinoma-bearing mice. Integr Cancer Ther 2007;6:66-73.

61. Soo HL, Quah SY, Sulaiman I, et al. Advances and challenges in developing andrographolide and its analogues as cancer therapeutic agents. Drug Discov Today 2019;24:1890-8.

62. Iruretagoyena MI, Tobar JA, González PA, et al. Andrographolide interferes with $\mathrm{T}$ cell activation and reduces experimental autoimmune encephalomyelitis in the mouse. J Pharmacol Exp Ther 2005;312:366-72.

63. Iruretagoyena MI, Sepúlveda SE, Lezana JP, et al. Inhibition of nuclear factor-kappa B enhances the capacity of immature dendritic cells to induce antigen-specific tolerance in experimental autoimmune encephalomyelitis. J Pharmacol Exp Ther 2006;318:59-67.

64. Naik SR, Hule A. Evaluation of immunomodulatory activity of an extract of andrographolides from Andographis paniculata. Planta Med 2009;75:785-91.

65. Wang W, Wang J, Dong SF, et al. Immunomodulatory activity of andrographolide on macrophage activation and specific antibody response. Acta Pharmacol Sin 2010;31:191-201.

66. Nie X, Chen SR, Wang K, et al. Attenuation of Innate Immunity by Andrographolide Derivatives Through NF-кB Signaling Pathway. Sci Rep 2017;7:4738.

67. Xu Y, Chen A, Fry S, et al. Modulation of immune response in mice immunised with an inactivated Salmonella vaccine and gavaged with Andrographis paniculata extract or andrographolide. Int Immunopharmacol 2007;7:515-23.

68. Ciampi E, Uribe-San-Martin R, Cárcamo C, et al. Efficacy of andrographolide in not active progressive multiple sclerosis: a prospective exploratory doubleblind, parallel-group, randomized, placebo-controlled trial. BMC Neurol 2020;20:173.

69. Das S, Mishra KP, Ganju L, et al. Andrographolide - A promising therapeutic agent, negatively regulates glial cell derived neurodegeneration of prefrontal cortex, hippocampus and working memory impairment. J Neuroimmunol 2017;313:161-75.

70. Yen TL, Chen RJ, Jayakumar T, et al. Andrographolide stimulates p38 mitogen-activated protein kinasenuclear factor erythroid-2-related factor 2-heme oxygenase 1 signaling in primary cerebral endothelial cells for definite protection against ischemic stroke in rats. Transl Res 2016;170:57-72.

71. Lu J, Ma Y, Wu J, et al. A review for the neuroprotective effects of andrographolide in the central nervous system. Biomed Pharmacother 2019;117:109078.

72. Zhang Z, Jiang J, Yu P, et al. Hypoglycemic and beta cell protective effects of andrographolide analogue for diabetes treatment. J Transl Med 2009;7:62.

73. Yu BC, Hung CR, Chen WC, et al. Antihyperglycemic effect of andrographolide in streptozotocin-induced diabetic rats. Planta Med 2003;69:1075-9.

74. Zhang C, Gui L, Xu Y, et al. Preventive effects of andrographolide on the development of diabetes in autoimmune diabetic NOD mice by inducing immune tolerance. Int Immunopharmacol 2013;16:451-6.

75. Peng S, Gao J, Liu W, et al. Andrographolide ameliorates OVA-induced lung injury in mice by suppressing ROS-mediated NF- $\mathrm{NB}$ signaling and NLRP3 inflammasome activation. Oncotarget 
2016;7:80262-74.

76. Gao F, Liu X, Shen Z, et al. Andrographolide Sulfonate Attenuates Acute Lung Injury by Reducing Expression of Myeloperoxidase and Neutrophil-Derived Proteases in Mice. Front Physiol 2018;9:939.

77. Hidalgo MA, Hancke JL, Bertoglio JC, et al. Andrographoline a new potential drug for the longterm treatment of rheumatoid arthritis disease. In: Matsuno H, editor. Innovative Rheumatology. Rijeka,

Cite this article as: Vetvicka V, Vannucci L. Biological properties of andrographolide, an active ingredient of Andrographis Paniculata: a narrative review. Ann Transl Med 2021;9(14):1186. doi: 10.21037/atm-20-7830
Croatia: InTech; 2013. p. 247-70.

78. Yu Z, Lu B, Sheng Y, et al. Andrographolide ameliorates diabetic retinopathy by inhibiting retinal angiogenesis and inflammation. Biochim Biophys Acta 2015;1850:824-31.

79. Dai Y, Chen SR, Chai L, et al. Overview of pharmacological activities of Andrographis paniculata and its major compound andrographolide. Crit Rev Food Sci Nutr 2019;59:S17-29. 